\title{
Evidence is limited about effective interventions for maintaining healthy peri-implant tissues
}

\section{What is the most effective intervention for maintaining healthy tissue around dental implants?}

\author{
Esposito M, Worthington HV, Coulthard P, Jokstad A. \\ Interventions for replacing missing teeth: maintaining and \\ re-establishing healthy tissues around dental implants \\ (Cochrane Review). The Cochrane Library 2002; 2002; Issue 3. \\ Oxford: Update Software
}

Data sources Studies were identified through the Cochrane Oral Health Group Specialised Register, the Cochrane Controlled Trials Register, Medline and EMbase, hand searches of dental journals, as well as contact with authors of all identified trials, 55 oral implant manufacturers and from two extensive personal libraries (ME and $A J)$ ). Study selection Randomised controlled trials (RCT) of oral implants that compared agents or interventions for maintaining or re-establishing healthy tissues around dental implants.

Data extraction and synthesis Data were independently extracted in duplicate by two reviewers (ME and HW). Authors were contacted for details of randomisation and withdrawals, and a quality assessment was carried out. The Cochrane Oral Health Group's statistical guidelines were followed.

Results Nine RCTs were identified. Five of these trials, which reported results from a total of 127 patients, were suitable for inclusion.

Conclusions There is only a little reliable evidence for deciding which are the most effective interventions for maintaining health around periimplant tissues. There was no evidence that the use of powered or sonic toothbrushes was superior to manual toothbrushing. There is a weak evidence that Listerine (Pfizer) mouthwash used twice a day for $30 \mathrm{~s}$, as an adjunct to routine oral hygiene, is effective in reducing plaque formation and marginal bleeding around implants. There was no evidence that phosphoric etching gel offered any clinical advantage over mechanical debridement. These findings are based on RCT that had short follow-up periods and few subjects. There is no reliable evidence for the most effective regimens for long-term maintenance. For the treatment of failing implants (peri-implantitis) there is no reliable evidence for preferring one therapeutic regimen over another. More RCT should be conducted in this area. In particular, there is a clear need for trials that investigate the most effective approach for the treatment of peri-implantitis and for trials with longer follow-up for maintenance. Such trials should be reported according the CONSORT guidelines (http://www.consort-statement.org/).

\section{Commentary}

The use of implants in dentistry is increasing. A greater understanding of the principles of oseo-integration and a wider range of techniques has contributed to the expansion of this treatment. Despite the increase in their use, however, it should still be recognised that implants are part of the oral environment and thus susceptible to failure (peri-implantitis) if the supporting tissues are not maintained in a healthy state. The present Cochrane Review is both timely and pertinent in the present implant culture.

In comparison with periodontal therapies, it is surprising that only nine RCT have been carried out to evaluate treatments for maintaining health around peri-implant tissues. Perhaps even more disconcerting is the lack of investigations into the management of peri-implantitis. Although the rate of failure is low, the consequences of a failing implant can be both costly and disastrous.

This review provides the appropriate background about the need to maintain implant health. As mentioned above, only a small number of RCT have been completed on the evaluation of different interventions to maintain implant health and these studies have been critically reviewed.

The shortcoming is the somewhat limited follow-up period (5 months or less) and the small number of subjects. Some defined criteria need to be applied to maintaining the health of implants, akin to the guidelines produced by the American Dental Association on therapeutic measures to evaluate products in the management of periodontal disease. Criteria such as follow-up period (minimum of 6 months) and number of subjects investigated should be adhered to. It is also apparent from the review that a number of outcome measures have been employed to evaluate the various interventions. Again, consistency in the measures used to evaluate plaque and inflammation would allow for more meaningful comparisons. Despite these technicalities the authors have managed to carry out a useful systematic review, although it probably raises more questions than solutions.

\section{Practice points}

- There is only a little reliable evidence about what are the most effective interventions for maintaining health around peri-implant tissues: more research is required.

- There was no evidence that powered brushes were better than manual brushing but there was weak evidence to support adjunctive use of Listerine mouthwash.

\section{Robin Seymour}

School of Dental Sciences, Newcastle Dental School, Newcastleupon-Tyne, UK

Evidence-Based Dentistry (2003) 4, 13.

doi:10.1038/sj.ebd. 6400168 